\title{
Influencia de la Construcción del Sistema de Recirculación de los Gases sobre la Formación de NOx en un Generador de Vapor de 350 MW
}

\author{
Georgiy Polupan $^{(1)}$, Guillermo Jarquin ${ }^{\left(2^{\star}\right)}$, Ignacio Carvajal ${ }^{(1)}$ y Juan A. Jiménez ${ }^{(3)}$ \\ (1)Instituto Politécnico Nacional, SEPI-ESIME-Zacatenco, Av. IPN s/n, Edif.5, UPALM, Colonia \\ Lindavista, 07738. México, D.F. (e-mail: gpolupan@ipn.mx, icarvajal@ipn.mx) \\ (2)Instituto Politécnico Nacional, SEPI-ESIME-Culhuacan, Av. Santa Ana No. 1000, Edif.2,tercer \\ piso, Colonia San Francisco Culhuacan, Coyoacán,C.P. 04430. México, D.F. (e-mail: \\ gjarquin@ipn.mx) \\ (3)Universidad Autónoma del Estado de México. UAP - Nezahualcóyotl. Av. Bordo de Xochiaca \\ s/n Col. Benito Juárez, 57000 Cd. Nezahualcóyotl. Edo. de México. (e-mail: \\ jjimenez@uaemex.mx)
}

*autor a quien se debe dirigir la correspondencia

Recibido Jun. 08, 2010; Aceptado Ago. 12, 2010; Versión Final recibida Oct. 11, 2010

\section{Resumen}

Se presentan los resultados de un estudio sobre la formación de NOx en un generador de vapor de $350 \mathrm{MW}$ que quema combustóleo. El objetivo de este trabajo fue determinar la formación de NOx utilizando dos construcciones diferentes de recirculación de los gases. La primera construcción consiste en introducir los gases de recirculación en la parte inferior del horno y la segunda en inyectar los gases de recirculación en el ducto de aire caliente. Para ambos casos el estudio se realiza a diferentes cargas térmicas, manteniendo constantes las fracciones de recirculación de gases para cada carga. Los resultados con la introducción de gases de recirculación en el ducto de aire caliente indican que la formación NOx fue hasta un 58 \% menor a los obtenidos con el sistema tradicional de inyección de gases en el fondo del horno.

Palabras clave: generador de vapor, sistema de recirculación, formación de NOx, combustóleo

\section{Effect About Construction of the Recirculation System on NOx Formation in a Boiler with Power of 350 MW}

\begin{abstract}
The paper presents the results of a study on the formation of NOx in a $350 \mathrm{MW}$ steam generator that burns fuel oil. The objective of this work was to determine the NOx formation applying two different constructions of recirculation gases. The first construction has the introduction of recirculation gases in the bottom of the furnace and the second one involves injection of recirculation gases in the hot air duct. In both cases, the study was developed at different thermal loads, maintaining constant the fractions of recirculation gases for each thermal load. The results show that the introduction of recirculation gases in the hot air duct reduces NOx formation by $58 \%$ compared to those obtained with the traditional method of injection of gases in the bottom of the furnace.
\end{abstract}

Keywords: steam generator, recirculation system, formation of NOx, fuel oil 


\section{INTRODUCCIÓN}

La formación de los óxidos de nitrógeno NOx ocurre debido a la oxidación del nitrógeno contenido en el aire y en el combustible. Los óxidos de nitrógeno $\left(\mathrm{NO}_{\mathrm{x}}\right)$, representan la suma de monóxido de nitrógeno (NO), dióxido de nitrógeno $\left(\mathrm{NO}_{2}\right)$ y óxido nitroso $\left(\mathrm{N}_{2} \mathrm{O}\right)$. La cantidad de $\mathrm{NO}_{2}$ y $\mathrm{N}_{2} \mathrm{O}$ no exceden $2 \%$ (Kotler, 1987). Existen tres mecanismos de formación de NOx: térmicos ( $\left.\mathrm{NO}_{\text {term }}\right)$, rápidos $\left(\mathrm{NO}_{\text {rapidos }}\right)$ y de combustible $\left(\mathrm{NO}_{\text {comb }}\right)$. La formación de $\mathrm{NO}_{\text {term, y }} \mathrm{NO}_{\text {rapidos }}$ se deben al nitrógeno contenido en el aire, y los NOx de combustible se originan del nitrógeno contenido en la composición química del combustible. (Zeldovich, 1947) fue el primero en explicar el mecanismo de formación de los NO térmicos. Las reacciones de la formación de $\mathrm{NO}_{\text {term }}$ están caracterizadas por la alta energía de activación. Los NOx térmicos se generan a altas temperaturas, superiores a $1800 \mathrm{~K}$ (Bartok et al., 1971).

Investigaciones recientes (Zheng et al., 2009) empleando algoritmos genéticos han mostrado una estrecha relación entre la emisión de NOx y los parámetros de operación de generadores de vapor; que con modificarlos se puede optimizar la combustión, para tener emisiones de NOx aceptables por la normatividad y evitando la instalación de equipos de tratamiento de gases costosos como los SCR y SNCR. (Havlena et al., 2005) aplica un modelo predictivo avanzado del control de la combustión planteando optimizar el exceso de aire que entra a la cámara de combustión del generador de vapor logrando el incremento de la eficiencia y la reducción de la formación de NOx. (Mafra M. R., et al., 2010) presenta un análisis experimental de la caracterización de la formación de $\mathrm{NO}_{\text {term }}$ y $\mathrm{NO}_{\text {rapidos }}$ en una cámara de combustión a diferentes relaciones de equivalencia. (Habib et al., 2008) presenta una simulación numérica del estudio de la formación de NOx en un generador de vapor de 160 MW quemando gas, mostrando la dependencia de la formación de NOx de la temperatura máxima, la temperatura promedio en la ZCA, así como de la influencia del exceso de aire y la posición de los quemadores. (Kuprianov et al., 2010) muestra la influencia de los parámetros de operación como técnica para controlar la emisión de NOx en una cámara de combustión de lecho fluidizado. Otras investigaciones realizadas por (Mahmoudi S., et al., 2010) se han dirigido a el estudio de tecnologías de control de NOx durante la postcombustión como es la reducción catalítica selectiva (SCR) y aplicando aditivos o reducción catalítica no selectiva (SNCR), el empleo de técnicas como la Oxi-fuel control (Tiggesa et al., 2009) que consiste en realizar la combustión próxima a la estequiometria en la presencia de aire puro $\left(95-99.5 \% \mathrm{~mol}_{2}\right.$ ) y otros más a la optimización de la emisión de NOx durante la combustión, empleando técnicas de análisis numéricos, simulación y redes neuronales (Zhou et al., 2004).

La formación de NOx en los generadores de vapor depende de cuatro parámetros fundamentales: temperatura promedio $\left(\bar{T}_{Z C A}\right)$ en la zona de combustión activa (ZCA), la densidad de flujo de calor reflejado $\left(q_{Z C A}^{r e f l}\right)$, el coeficiente de exceso de aire $\left(\alpha_{Z C A}\right)$ y el tiempo de residencia de los gases en la zona de combustión activa ( $\left.{ }^{2} z \mathrm{ZCA}\right)$ (Roslyakov et al, 1997). El tiempo en que se estabiliza la concentración de NOx, en un rango de temperatura de 1800 a $1900 \mathrm{~K}$ es de 4 a 20 segundos aproximadamente, en los hornos de los generadores de vapor, el tiempo de residencia de los gases en la zona de combustión es menor (Kotler, 1987). La concentración de NOx en dependencia del tiempo de residencia para el rango de valores de 4 a 20 segundos, ya ha sido determinada (Roslyakov et al, 1997) mostrando una dependencia lineal, al incrementar el tiempo de residencia de los productos de la combustión en la ZCA, se incrementa proporcionalmente la formación de NOx. Por otro lado, el coeficiente de exceso de aire sobre la formación de NOx (Fenimore, 1971) muestra un comportamiento de un polinomio de cuarto grado y tiene valores máximos, para el rango de excesos de aire de $\alpha=1.10 \ldots 1.25$, al quemar gas natural $y$ combustóleo.

\section{METODOLOGÍA}

La formación de NOx en los hornos de los generadores de vapor depende principalmente de: la temperatura máxima del horno, el coeficiente de exceso de aire y el tiempo de residencia. 
Actualmente no existen métodos analíticos para determinar la temperatura máxima del horno, por lo consiguiente, se usan parámetros que si se pueden calcular y que representan a la temperatura máxima. Estos parámetros son: la temperatura promedio del horno y la densidad de flujo de calor reflejado.

La ecuación para el cálculo de la concentración de NOx (en ppm) en los productos de combustión en la salida de la Zona de Combustión Activa al quemar combustóleo está dado por (Roslyakov, et al., 1997):

$$
\begin{aligned}
& C_{N O_{x}}=\left[24.3 \exp \left(0.19 \frac{\bar{T}_{Z C A}-1650}{100}\right)-12.3\right] x\left[\exp \left(q_{Z C A}^{r e f l}-1\right)\right] x\left[15.1+2.8\left(\alpha_{Z C A}-1.09\right)\right. \\
& \left.+73\left(\alpha_{Z C A}-1.09\right)^{2}+72.3\left(\alpha_{Z C A}-1.09\right)^{3}-131.7\left(\alpha_{Z C A}-1.09\right)^{4}\right] x \tau_{Z C A}
\end{aligned}
$$

La temperatura promedio de los productos de la combustión en la ZCA se calcula:

$$
\bar{T}_{Z C A}=T_{a d}^{s}\left(1 \quad \bar{T}_{Z C A}\right)^{0.25}\left(1-R^{1+n * R^{2}}\right)
$$

En la ecuación (2) $n$ es el valor empírico que depende del lugar de introducción de los gases de recirculación; $n=6.5$ inyección de gases de recirculación por la parte inferior del horno del generador de vapor y $n=3.0$ para la introducción de gases de recirculación en el ducto de aire caliente (Roslyakov, et al. 2001).

El factor de eficiencia térmica de superficie en ZCA se determina con:

$$
\bar{\Psi}_{Z C A}=\frac{\boldsymbol{\Sigma} \Psi_{i} A_{i}}{\boldsymbol{\Sigma} A_{i}}
$$

Siendo $A_{i}$ y $\Psi_{i}$ respectivamente: áreas de superficies de la ZCA y sus eficiencias térmicas.

La temperatura adiabática de la combustión incompleta del combustible $T_{a\rfloor}^{r}(K)$ se calcula considerando que el calor disponible del horno $Q_{\text {disp }}$ es transformado directamente en entalpía de gases de combustión:

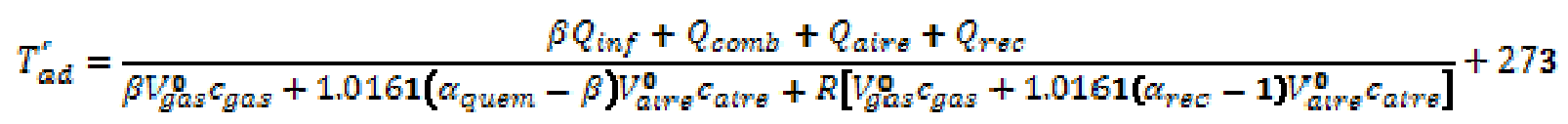

En la ecuación (4) la cantidad teórica de aire que es necesario para la combustión completa del combustible para el quemado del combustóleo es:

$V_{\text {air }}^{0}=0.0889(C+0.375 S)+0.265 H-0.03330$

Y el volumen de los gases de combustión producidos en la quema de $1 \mathrm{~kg}$ de combustible es:

$V_{g a g}^{O}=V_{R O z}+V_{N z}^{0}+V_{H z O}+(\alpha-1) V_{a i r e}^{0}$

Hasta aquí se han presentado las variables que intervienen para el cálculo de la temperatura promedio.

A continuación el flujo de calor reflejado de la ZCA se obtiene de (Roslyakov, et al. 1997):

$q_{Z E A}^{r e f l}=q_{Z c A}\left(1-\bar{\Psi}_{Z C A}\right)$

El flujo de calor $q_{z c s}$ en la zona de combustión activa se determina por la siguiente ecuación: 
$q_{z C A}=\frac{B\left(\beta Q_{i n f}+Q_{\text {comb }}+Q_{\text {aire }}+Q_{r \in c}\right)}{A_{z C A}}$

El coeficiente de exceso de aire, es determinado con la suma del exceso de aire del horno $\left(\alpha_{\text {ivgas. }}\right)$ y el aire que es introducido por los gases de recirculación $\alpha_{r e c}$.

$\alpha_{Z \Xi A}=\alpha_{\text {hogar }}+R\left[\alpha_{r e s}-1\right]$

El tiempo de residencia de los productos de la combustión en la ZCA, se determina:

$\tau_{Z C A}=\frac{a b c_{Z C A}^{R} \xi}{B V_{g a S}^{R}\left(T_{Z C A} / 273\right)}$

En la ecuación (10) $\xi$ - factor de llenado de la ZCA por gases de combustión, determinado en (Bezgreshnov, 1991) con el valor de $\mathbf{0 . 7 0}$ para hornos que tienen quemadores tangenciales.

El volumen de los productos de la combustión en la zona de combustión activa incluyendo los gases de recirculación se determina por la ecuación:

$V_{g a s}^{R}=\beta V_{g a s}^{0}+1.0161\left(\alpha_{\text {quem }}-\beta\right) V_{a i r g}^{0}+R\left[V_{g a s}^{0}+1.0161\left(\alpha_{r a t}-1\right) V_{a i r g}^{0}\right]$

La altura de la zona de combustión activa se calcula mediante la ecuación:

$c_{Z C A}^{R}=\varepsilon_{Z C A} \frac{V_{Z C A}^{R}}{V_{Z C A}}$

EL tiempo de residencia es el cuarto parámetro para la determinación de NOx en el polinomio experimental.

\section{RESULTADOS}

La tabla 1 presenta los parámetros fundamentales de la formación de óxidos de Nitrógeno y la concentración de NOx para los dos diferentes sistemas de recirculación.

Tabla 1: Resultados de los parámetros de la ZCA y concentración de NOx.

\begin{tabular}{|c|c|c|c|c|c|}
\hline \multirow{2}{*}{ Parámetro } & \multirow{2}{*}{ Unidad } & \multicolumn{4}{|c|}{ Carga Térmica } \\
\hline & & $100 \%$ & $75 \%$ & $50 \%$ & $25 \%$ \\
\hline$R$ (Fracción de gases de recirculación) & - & 0.23 & 0.44 & 0.62 & 0.54 \\
\hline $\bar{T}_{Z C A} \quad$ Para $n=\mathbf{3 . 0}$ & $K$ & 2007.8 & 1893.0 & 1579.4 & 1527.2 \\
\hline$\overline{\mathbf{T}}_{\mathrm{ZCA}} \quad$ Para $\mathrm{n}=6.5$ & $K$ & 2134.5 & 2130.6 & 1928.1 & 1788.2 \\
\hline$a_{Z C A}$ & - & 1.062 & 1.072 & 1.243 & 1.462 \\
\hline$q_{Z C A}^{r a f}$ & $M W_{h_{n}}=$ & 0.810 & 0.617 & 0.458 & 0.215 \\
\hline$\tau_{Z E A} ; n=3.0$ & $s$ & 0.470 & 0.666 & 1.10 & 2.36 \\
\hline$\tau_{Z Z A} ; n=6.5$ & $s$ & 0.442 & 0.592 & 0.899 & 2.01 \\
\hline$C_{N O_{x}} \operatorname{con} n=6.5$ & ppm & 405 & 368 & 263 & 255 \\
\hline$C_{N O_{x}} \operatorname{con} n=3.0$ & ppm & 315 & 225 & 99 & 108 \\
\hline Mitigación de emisiones & $\%$ & 22.3 & 38.8 & 62.3 & 58.9 \\
\hline
\end{tabular}




\section{CONCLUSIONES}

La metodología presentada muestra que es más efectivo inyectar los gases de recirculación en el ducto de aire caliente que introducirlos en el fondo del horno del generador de vapor, debido a que se influye directamente en los parámetros de la ZCA principalmente en la temperatura promedio. Se mitiga la formación de NOx en $22.3 \%$ desde 405.0 ppm hasta $315.2 \mathrm{ppm}$ con carga térmica de $100 \%$ y se disminuye $58.9 \%$ desde 255 ppm hasta $107.5 \mathrm{ppm}$ a carga térmica de $25 \%$. Las concentraciones de NOx calculadas usando el método desarrollado difieren hasta un máximo de $15 \%$ de las mediciones experimentales.

\section{NOMENCLATURA}

\begin{tabular}{|c|c|c|}
\hline$a$ & ancho del hogar & \\
\hline$b$ & profundidad del hogar & \\
\hline 8 & consumo de combustible & \\
\hline aire & capacidad calorífica del aire & \\
\hline$c_{Z C A}^{R}$ & altura de la zona de combustión activa & \\
\hline$c_{g a g}$ & capacidad calorífica de los productos de la combustión & \\
\hline $\mathrm{C}_{\mathrm{NO}_{\mathrm{X}}}$ & concentración de óxidos de nitrógeno & {$[p$} \\
\hline $\begin{array}{l}n \\
q z C_{A}\end{array}$ & $\begin{array}{l}\text { valor correspondiente al lugar de entrada de los gases de recirculación } \\
\text { densidad de flujo de calor en la zona de combustión activa }\end{array}$ & \\
\hline$q_{Z C A}^{m e f l}$ & flujo de calor reflejado en la ZCA & \\
\hline Qaire & calor suministrado con el aire caliente en ZCA & \\
\hline Roomb & calor sensible introducido en la ZCA & \\
\hline$Q_{\text {infl }}$ & poder calorífico inferior del combustible & \\
\hline Qrac & calor suministrado con los gases de recirculación & \\
\hline 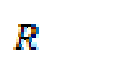 & fracción de gases de recirculación & {$[-]$} \\
\hline $\bar{T}_{Z C A}$ & temperatura promedio de los gases en la ZCA & {$[K]$} \\
\hline$T_{a d}^{s}$ & temperatura adiabática de combustión incompleta & \\
\hline$V_{a t r e}^{0}$ & volumen teórico de aire para quemar $1 \mathrm{~kg}$ de combustible & \\
\hline$V_{g a s}^{R}$ & volumen de los productos de la combustión, incluyendo recirculación & \\
\hline$V_{\text {gas }}^{0}$ & volumen teórico de los productos de la combustión & $m^{3} / h$ \\
\hline$\varepsilon_{Z Z A}$ & coeficiente de exceso de aire en la ZCA & {$[-]$} \\
\hline$\alpha_{\text {quem }}$ & coeficiente de exceso de aire en el quemador & {$[-]$} \\
\hline$\alpha_{\operatorname{rec}}$ & $\begin{array}{l}\text { coeficiente de exceso de aire considerando gases de recirculación } \\
\text { eficiencia de combustión }\end{array}$ & {$[-]$} \\
\hline$\Psi_{i}$ & coeficiente de eficiencia térmica de las paredes de agua & {$[-]$} \\
\hline $\bar{\varphi}_{z C A}$ & coeficiente de eficiencia térmica promedio de paredes de agua & {$[-]$} \\
\hline & coeficiente de llenado de la ZCA con gases de combustión & {$[-]$} \\
\hline & tiempo de residencia de los gases en la ZCA & {$[s]$} \\
\hline
\end{tabular}




\section{REFERENCIAS}

Bartok W., Engleman V., Laboratory studies and mathematical modeling of NOx formation in combustion processes, ESSO Research and engineering Company. Final report. Contract CPA 70-90. Linden. New-Jersey, GRV.3GNOS.71.

Bezgreshnov A.N., Lipov Yu. M. y Shleyfer B.M., Calculation of steam boilers, Moscow, Energoatomizdat, (1991).

CKTI-VTI, Thermal design for power boilers Standard Method, 259, Sankt-Petersburg, Rusia (1998).

Fenimore C.P. Formation of nitric oxide in premixed hydrocarbon flame. Proc. of 13-th International Symposium on Combustion, p.373 (1971)

Habib M.A., Elshafei M., Dajani M., Influence of combustion parameters on NOx production in an industrial boiler, Computers \& Fluids, 37, 12-23 (2008).

Havlena Vladimir, Jiri Findejs, Application of model predictive control to advanced combustion control, Control Engineering Practice, 13, 671-680 (2005).

Jiménez García J. A. Análisis de la formación de NOx en el generador de vapor de 350 MW quemando combustóleo, Tesis de Maestría, Ingeniería Mecánica, Instituto Politécnico Nacional, México (2008).

Kotler V.R., Nitrogen oxides in boiler smock gases, Moscow, Energoatomizdat, (1987).

Kuprianov Vladimir I., Kaewklum Rachadaporn, Chakritthakul Songpol, Effects of operating conditions and fuel properties on emission performance and combustion efficiency of a swirling fluidized-bed combustor fired with a biomass fuel, Energy, XXX, 1-11 (2010).

Mafra M. R., Fassani F. L., Zanoelo E. F., Bizzo W. A. Influence of swirl number and fuel equivalence ratio on NO emissions in experimental LPG fired chamber. Applied Thermal Engineering (30) 8-9, 928934 (2010).

Mahmoudi S., Baeyens J., Sevilla J. P. K. NOx formation in the selective non catalytic reduction (SNCR) in the fluidized bed combustor of biomass. Biomass and bioenergy (34). Págs. 1393-1409 (2010)

Roslyakov P.V. y Egorova L.E., Method of calculation of nitrogen oxide formation in hot water and steam boilers. Thermal Engineering, 4, 67-74 (1997).

Roslyakov P.V. y Zakirov I.A., Nonstoichiometric burning of natural gas and boiler oil on Power Plants. Moscow, MEI, (2001).

Tiggesa K.-D., y otros 11 autores, Conversion of existing coal-fired power plants to oxyfuel combustion: case study with experimental results and CFD simulations, Energy Procedia, 1, 549-546 (2008).

Zeldovich Ya., Sadovnikiv I. y Frank-Kameneckiy D, Nitrogen oxidation at burning. Leningrad, USSR AC, (1947).

Zheng Li-Gang, Zhou Hao, Cen Ke-Fa, Wang Chun-Lin, A comparative study of optimization algorithms for low NOx combustion modification at a coal-fired utility boiler, Expert Systems with Applications, 36, 2780-2793 (2009).

Zhou Hao , Cen Kefa, Fan Jianren, Modeling and optimization of the NOx emission characteristics of a tangentially fired boiler with artificial neural networks, Control Engineering Practice, 12, 707-723 (2004). 